\title{
Some Characterizations of Constant Ratio Curves According to Type-2 Bishop Frame in Euclidean 3-space $E^{3}$
}

\author{
Hülya Gün Bozok* Sezin Aykurt Sepet and Mahmut Ergüt \\ (Communicated by Ion Mihai)
}

\begin{abstract}
In this paper, we study a twisted curve in the 3-dimensional Euclidean space $E^{3}$ as a curve whose position vector can be determined as linear combination of its type-2 Bishop frame. We research these curves according to their curvature functions. Moreover we obtain some results of $T$-constant and $N$-constant type curves in the 3-dimensional Euclidean space $E^{3}$.
\end{abstract}

Keywords: Position vector; type-2 Bishop frame; constant ratio curves.

AMS Subject Classification (2010): 53A04

\section{Introduction}

The theory of curves has an important role in differential geometry. One of these curves is twisted curve, a curve $x: I \subset \mathbb{R} \rightarrow E^{3}$ which has non-zero Frenet curvatures $k_{1}(s)$ and $k_{2}(s)$ is called twisted curve. For a regular curve $x(s)$, the position vector of $x$ can be decompose into its tangential and normal components at each point:

$$
x=x^{T}+x^{N} .
$$

A curve $x(s)$ with $k_{1}(s)>0$ is called constant ratio if the ratio $\left\|x^{T}\right\|:\left\|x^{N}\right\|$ is constant on $x(I)$. Here $\left\|x^{T}\right\|$ and $\left\|x^{N}\right\|$ denote the length of $x^{T}$ and $x^{N}$, respectively [4]. A curve in $E^{n}$ is said to be $T$-constant (resp. $N$-constant) if the tangential component $x^{T}$ (resp. the normal component $x^{N}$ ) of its position vector $x$ is of constant length [4]. In recent years constant ratio curves are studied in Euclidean and Minkowski space [7, 3, 8, 2].

On the other hand, L.R. Bishop defined Bishop frame, which is known alternative or parallel frame of the curves with the help of parallel vector fields [1]. Then, S. Yilmaz and M. Turgut introduced a new version of the Bishop frame which is called type-2 Bishop frame [10]. Thereafter, E. Ozyilmaz studied classical differential geometry of curves according to type-2 Bishop trihedra [9].

In this study we researched a twisted curve in the 3-dimensional Euclidean space $E^{3}$ as a curve whose position vector satisfies the following parametric equation

$$
x(s)=\lambda(s) N_{1}(s)+\mu(s) N_{2}(s)+\gamma(s) B(s)
$$

where $\lambda, \mu, \gamma$ are differentiable functions and $\left\{N_{1}, N_{2}, B\right\}$ is its type-2 Bishop frame. We characterize these curves according to their curvature functions. Moreover we obtain some results of $T$-constant and $N$-constant type curves in the 3-dimensional Euclidean space $E^{3}$.

\section{Preliminaries}

The standard flat metric of 3-dimensional Euclidean space $E^{3}$ is given by

$$
\langle,\rangle: d x_{1}^{2}+d x_{2}^{2}+d x_{3}^{2}
$$


where $\left(x_{1}, x_{2}, x_{3}\right)$ is a rectangular coordinate system of $E^{3}$. For an arbitrary vector $x$ in $E^{3}$, the norm of this vector is defined by $\|x\|=\sqrt{\langle x, x\rangle}$. $\alpha$ is called a unit speed curve, if $\left\langle\alpha^{\prime}, \alpha^{\prime}\right\rangle=1$. Suppose that $\{t, n, b\}$ is the moving Frenet-Serret frame along the curve $\alpha$ in $E^{3}$. For a unit speed curve $\alpha$, the Frenet-Serret formulae can be given as

$$
\begin{aligned}
t^{\prime} & =\kappa n \\
n^{\prime} & =-\kappa t+\tau b \\
b^{\prime} & =-\tau n
\end{aligned}
$$

where

$$
\begin{aligned}
& \langle t, t\rangle=\langle n, n\rangle=\langle b, b\rangle=1, \\
& \langle t, n\rangle=\langle t, b\rangle=\langle n, b\rangle=0 .
\end{aligned}
$$

and here, $\kappa=\kappa(s)=\left\|t^{\prime}(s)\right\|$ and $\tau=\tau(s)=-\left\langle n, b^{\prime}\right\rangle$. Furthermore, the torsion of the curve $\alpha$ can be given

$$
\tau=\frac{\left[\alpha^{\prime}, \alpha^{\prime \prime}, \alpha^{\prime \prime \prime}\right]}{\kappa^{2}} .
$$

Along the paper, we assume that $\kappa \neq 0$ and $\tau \neq 0$.

Bishop frame is an alternative approachment to define a moving frame. Assume that $\alpha(s)$ is a unit speed regular curve in $E^{3}$. The type-2 Bishop frame of the $\alpha(s)$ is expressed as [10]

$$
\begin{aligned}
N_{1}^{\prime} & =-k_{1} B \\
N_{2}^{\prime} & =-k_{2} B \\
B^{\prime} & =k_{1} N_{1}+k_{2} N_{2} .
\end{aligned}
$$

The relation matrix may be expressed as

$$
\left[\begin{array}{l}
t \\
n \\
b
\end{array}\right]=\left[\begin{array}{ccc}
\sin \theta(s) & -\cos \theta(s) & 0 \\
\cos \theta(s) & \sin \theta(s) & 0 \\
0 & 0 & 1
\end{array}\right]\left[\begin{array}{l}
N_{1} \\
N_{2} \\
B
\end{array}\right] .
$$

where $\theta(s)=\int_{0}^{s} \kappa(s) d s$. Then, type-2 Bishop curvatures can be defined in the following

$$
\begin{gathered}
k_{1}(s)=-\tau(s) \cos \theta(s), \\
k_{2}(s)=-\tau(s) \sin \theta(s) .
\end{gathered}
$$

On the other hand,

$$
\theta^{\prime}=\kappa=\frac{\left(\frac{k_{2}}{k_{1}}\right)^{\prime}}{1+\left(\frac{k_{2}}{k_{1}}\right)^{2}} .
$$

The frame $\left\{N_{1}, N_{2}, B\right\}$ is properly oriented, $\tau$ and $\theta(s)=\int_{0}^{s} \kappa(s) d s$ are polar coordinates for the curve $\alpha$. Then, $\left\{N_{1}, N_{2}, B\right\}$ is called type-2 Bishop trihedra and $k_{1}, k_{2}$ are called type-2 Bishop curvatures.

\section{Constant Ratio Curves According to type-2 Bishop Frame}

Let $x(s)$ be a twisted curve whose position vector can be determined as linear combination of its type- 2 Bishop frame, then its position vector can be written as

$$
x(s)=\lambda(s) N_{1}(s)+\mu(s) N_{2}(s)+\gamma(s) B(s)
$$

where $\lambda, \mu, \gamma$ are differentiable functions and $\left\{N_{1}, N_{2}, B\right\}$ is its type-2 Bishop frame. Differentiating the equation (3.1) and using equation (2.3) we get

$$
\begin{aligned}
x^{\prime}(s) & =\left(\lambda^{\prime}+\gamma k_{1}\right) N_{1}(s)+\left(\mu^{\prime}+\gamma k_{2}\right) N_{2}(s) \\
& +\left(\gamma^{\prime}-\lambda k_{1}-\mu k_{2}\right) B(s)
\end{aligned}
$$


where $k_{1}(s)$ and $k_{2}(s)$ are Bishop curvatures. On the other hand if $N_{1}$ is taken instead of tangent vector, and considering above equation we have the following

$$
\begin{aligned}
\lambda^{\prime}+\gamma k_{1}-1 & =0 \\
\mu^{\prime}+\gamma k_{2} & =0 \\
\gamma^{\prime}-\lambda k_{1}-\mu k_{2} & =0 .
\end{aligned}
$$

Definition 3.1. Let $x: I \subset \mathbb{R} \rightarrow E^{n}$ be a unit speed curve in $E^{n}$. Then the position vector of $x$ can be decompose into its tangential and normal components at each point as

$$
x=x^{T}+x^{N}
$$

if the ratio $\left\|x^{T}\right\|:\left\|x^{N}\right\|$ is constant on $x(I)$ then $x$ is said to be constant ratio [4].

For a unit speed curve $x$ in $E^{n}$ the gradient of the distance function $\rho=\|x(s)\|$ is given by

$$
\operatorname{grad} \rho=\frac{d \rho}{d s} x^{\prime}(s)=\frac{\left\langle x(s), x^{\prime}(s)\right\rangle}{\|x(s)\|} x^{\prime}(s)
$$

where $T$ is the tangent vector of $x$. The following results can be given for constant ratio curves.

Theorem 3.1. [5] Let $x: I \subset \mathbb{R} \rightarrow E^{n}$ be a unit speed regular curve in $E^{n}$. Then $\|$ grad $\|=c$ holds for a constant $c$ if and only if the following three cases occurs:

(i) $x(I)$ is contained in a hypersphere centered at the origin.

(ii) $x(I)$ is an open portion of a line through the origin.

(iii) $x(s)=c s y(s), c \in(0,1)$, where $y=y(u)$ is a unit curve on the unit sphere of $E^{n}$ centered at the origin and $u=\frac{\sqrt{1-c^{2}}}{c} \ln s$.

Corollary 3.1. [5] Let $x: I \subset \mathbb{R} \rightarrow E^{n}$ be a unit speed regular curve in $E^{n}$. Then up to a translation of the arc length function $s$, we have

(i) $\|\operatorname{grad}\| \|=0 \Longleftrightarrow x(I)$ is contained in a hypersphere centered at the origin.

(ii) $\|\operatorname{grad}\|=1 \Longleftrightarrow x(I)$ is an open portion of a line through the origin.

(iii) $\|\operatorname{grad} \rho\|=c \Longleftrightarrow \rho=\|x(s)\|=$ cs for $c \in(0,1)$.

(iv) If $n=2$ and $\|\operatorname{grad} \rho\|=c$ for $c \in(0,1)$, then the curvature of $x$ satisfies

$$
\kappa^{2}=\frac{1-c^{2}}{c^{2} \sqrt{s^{2}+b}}
$$

for some real constant $b$.

For twisted curves according to type-2 Bishop frame in $E^{3}$ we get the following results.

Proposition 3.1. Let $x: I \subset \mathbb{R} \rightarrow E^{3}$ be a unit speed curve in $E^{3}$. If $x$ is a curve of constant ratio then its position vector can be written as

$$
\begin{aligned}
x(s) & =\left(c^{2} s\right) N_{1}(s)-\left[\frac{c^{2} s k_{1}}{k_{2}}+\frac{\left(1-c^{2}\right) k_{1}^{\prime}}{k_{1}^{2} k_{2}}\right] N_{1}(s) \\
& +\left(\frac{1-c^{2}}{k_{1}}\right) B(s)
\end{aligned}
$$

for some differential functions, $c \in(0,1)$. 
Proof. Let $x$ be a curve of constant ratio, then from corollary 3.1. the distance function of $x$ can be written as $\rho=\|x(s)\|=c s$ for some real constant $c$. Moreover considering (3.3) we have

$$
\|\operatorname{grad} \rho\|=\frac{\left\langle x(s), x^{\prime}(s)\right\rangle}{\|x(s)\|}
$$

Because of $x$ is a twisted curve of $E^{3}$, the equation (3.1) is satisfied. Then we get $\lambda=c^{2} s$. Therefore substituting $\lambda=c^{2} s$ in the equation (3.2) we obtain

$$
\begin{aligned}
& \mu=-\frac{c^{2} s k_{1}}{k_{2}}-\frac{\left(1-c^{2}\right) k_{1}^{\prime}}{k_{1}^{2} k_{2}}, \\
& \gamma=\frac{1-c^{2}}{k_{1}} .
\end{aligned}
$$

If we consider the above value of $\lambda, \mu, \gamma$ and substituting these value in equation (3.1) we obtain the equation (3.4) which complete the proof.

\section{T-Constant Curves}

Definition 4.1. Let $x: I \subset \mathbb{R} \rightarrow E^{n}$ be a unit speed curve in $E^{n}$. If $\left\|x^{T}\right\|$ is constant then $x$ is called a $T$-constant curve. For a $T$-constant curve $x$ either $\left\|x^{T}\right\|=0$ or $\left\|x^{T}\right\|=\eta$ for some non-zero smooth function $\eta$. Moreover, a $T$-constant curve $x$ is called first kind if $\left\|x^{T}\right\|=0$, otherwise second kind [6].

As a result of the equation (3.2), we obtain the following expression.

Theorem 4.1. Let $x: I \subset \mathbb{R} \rightarrow E^{3}$ be a unit speed twisted curve in $E^{3}$ that satisfies the equation (3.1). Then $x$ is a $T$-constant curve of first kind if and only if

$$
\frac{k_{2}}{k_{1}}-\left(\frac{k_{1}^{\prime}}{k_{1}^{2} k_{2}}\right)^{\prime}=0
$$

where $k_{1}, k_{2}$ are Bishop curvatures.

Proof. Suppose that $x$ is a $T$-constant curve of first kind. Then using the first and third equation of (3.2) we get

$$
\begin{aligned}
& \gamma=\frac{1}{k_{1}}, \\
& \mu=-\frac{k_{1}^{\prime}}{k_{1}^{2} k_{2}},
\end{aligned}
$$

where $k_{1}, k_{2}$ are Bishop curvatures. Substituting above equation into the second equation of (3.2) we obtain the desired result.

Theorem 4.2. Let $x: I \subset \mathbb{R} \rightarrow E^{3}$ be a unit speed twisted curve in $E^{3}$ that satisfies the equation (3.1). If $x$ is a $T$ constant curve of second kind then the position vector of the curve is given by

$$
x=\lambda N_{1}(s)-\left(\frac{\lambda k_{1}}{k_{2}}+\frac{k_{1}^{\prime}}{k_{1}^{2} k_{2}}\right) N_{2}(s)+\frac{1}{k_{1}} B(s)
$$

where $\lambda$ is a constant function.

Proof. Suppose that $x$ is a $T$-constant curve of second kind. Then using the equation (3.2) we have

$$
\gamma=\frac{1}{k_{1}}
$$

and considering the value of $\gamma$ in the third equation of (3.2) we obtain

$$
\mu=-\frac{k_{1}^{\prime}}{k_{1}^{2} k_{2}}-\frac{\lambda k_{1}}{k_{2}}
$$

where $\lambda$ is a constant function. So, substituting the value of $\mu, \gamma$ into the equation (3.1) we obtain the result. 
Corollary 4.1. Let $x: I \subset \mathbb{R} \rightarrow E^{3}$ be a unit speed twisted curve in $E^{3}$. If $x$ is a T-constant curve of second kind then the functions $\lambda, \mu, \gamma$ satisfied the following equation

$$
\gamma^{2}+\mu^{2}=2 \lambda s+c
$$

Proof. Suppose that $x$ is a $T$-constant curve of second kind. Then using the equation (3.2) we get

$$
\begin{aligned}
& k_{1}=\frac{1}{\gamma}, \\
& k_{2}=-\frac{\mu^{\prime}}{\gamma} .
\end{aligned}
$$

Then substiuting this values into the third equation of (3.2) we have the following differential equation

$$
\gamma \gamma^{\prime}+\mu \mu^{\prime}=\lambda
$$

which is the solution of (4.3).

\section{N-Constant Curves}

Definition 5.1. Let $x: I \subset \mathbb{R} \rightarrow E^{n}$ be a unit speed curve in $E^{n}$. If $\left\|x^{N}\right\|$ is constant then $x$ is called a $N$-constant curve. For a $N$-constant curve $x$ either $\left\|x^{N}\right\|=0$ or $\left\|x^{N}\right\|=\nu$ for some non-zero smooth function $\nu$. Moreover, a $N$-constant curve $x$ is called first kind if $\left\|x^{N}\right\|=0$, otherwise second kind [6].

For a $N$-constant curve $x$ the following equation satisfied

$$
\left\|x^{N}(s)\right\|^{2}=\mu^{2}(s)+\gamma^{2}(s)=\omega
$$

where $\omega$ is a constant function.

Considering the equation (3.1), (3.2) and (5.1) we obtain some results as follows.

Lemma 5.1. Let $x: I \subset \mathbb{R} \rightarrow E^{3}$ be a unit speed curve in $E^{3}$. Then $x$ is a $N$-constant curve if and only if

$$
\begin{aligned}
\lambda^{\prime} & =1-\gamma k_{1} \\
\mu^{\prime} & =-\gamma k_{2} \\
\gamma^{\prime} & =\lambda k_{1}+\mu k_{2} \\
0 & =\gamma \gamma^{\prime}+\mu \mu^{\prime}
\end{aligned}
$$

the above equation hold, where $\lambda(s), \mu(s), \gamma(s)$ are differentiable functions.

Proposition 5.1. Let $x: I \subset \mathbb{R} \rightarrow E^{3}$ be a unit speed curve in $E^{3}$. Then $x$ is a $N$-constant curve of first kind if and only if $x(I)$ is an open portion of a straight line [3].

Proof. Let $x$ is a $N$-constant curve in $E^{3}$, so the equation (5.1) holds. Moreover if $x$ is a $N$-constant curve of first kind then using (5.1) we have $\mu=\gamma=0$ which implies that $k_{1}=k_{2}=0$. So $x$ becomes a part of straight line.

Theorem 5.1. Let $x: I \subset \mathbb{R} \rightarrow E^{3}$ be a unit speed twisted curve in $E^{3}$. If $x$ is a $N$-constant curve of second kind then the curve has the following parametrization

$$
x(s)=\left(-\frac{k_{1}^{\prime}}{k_{1}^{2} k_{2}}\right) N_{2}(s)+\frac{1}{k_{1}} B(s)
$$

or

$$
x(s)=(s+a) N_{1}(s)+c N_{2}(s)
$$

where $a$ and $c$ are real constants. 
Proof. Suppose that $x$ is a $N$-constant curve of second kind then substituting the second and third equation of (5.2) into the last equation of (5.2) we have

$$
\begin{aligned}
\mu\left(-\gamma k_{2}\right)+\gamma\left(\lambda k_{1}+\mu k_{2}\right) & =0 \\
\gamma \lambda k_{1} & =0
\end{aligned}
$$

Since $k_{1} \neq 0$ we have two possibilities that $\lambda=0$ or $\gamma=0$. If $\lambda=0$ then $x$ is a $T$-constant curve and from the first and third equation of (5.2) $x$ has the following parametrization

$$
x(s)=\left(-\frac{k_{1}^{\prime}}{k_{1}^{2} k_{2}}\right) N_{2}(s)+\frac{1}{k_{1}} B(s) .
$$

If $\gamma=0$ then using the equation (5.2) we obtain

$$
\begin{aligned}
& \lambda^{\prime}=1 \\
& \mu^{\prime}=0
\end{aligned}
$$

Then $x$ satisfied the equation (5.4) which complete the proof.

\section{References}

[1] Bishop, L. R., There is more than one way to frame a curve. Am. Math. Monthly, 82 (1975), no.3, $246-251$.

[2] Büyükkütük, S. and Öztürk, G., Constant ratio curves according to parallel transport frame in Euclidean 4-space $E^{3}$. Adv. New Trends in Math. Sciences, 4 (2015), 171-178.

[3] Büyükkütük, S. and Öztürk, G., Constant ratio curves according to Bishop frame in Euclidean 3-space E $E^{3}$. Gen. Math. Notes, 28 (2015),no.1, 81-91.

[4] Chen, B. Y., Constant ratio hypersurfaces. Soochow J. Math., 28 (2001), 353-362.

[5] Chen, B. Y., More on convolution of Riemannian manifolds. Beitrage Algebra Geom., 44 (2003), no.1,9-24.

[6] Chen, B. Y., Geometry of position functions of Riemannian submanifolds in pseudo-Euclidean space. J. Geom., 74 (2002), 61-77.

[7] Gürpinar, S., Arslan, K. and Öztürk, G., A characterization of constant-ratio curves in Euclidean 3-space $E^{3}$. (2014), arXiv:1410.5577.

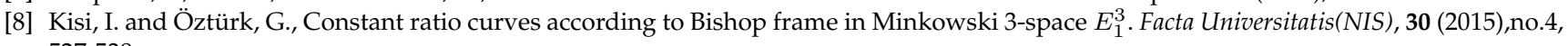
527-538.

[9] Ozyilmaz, E., Classical differential geometry of curves according to type-2 bishop trihedra. Mathematical and Computational Applications, 16 (2011),no.4, 858-867.

[10] Yilmaz, S. and Turgut, M., A new version of Bishop frame and an application to spherical images. J. Math. Anal. Appl., 371 (2010), $764-776$.

\section{Affiliations}

HÜLYA GÜN BOZOK

ADDRESS: Osmaniye Korkut Ata University, Department of Mathematics, 80000, Osmaniye, Turkey.

E-MAIL: hulyagun@osmaniye.edu.tr

ORCID ID : orcid.org/0000-0002-7370-5760

\section{SEZIN AYKURT SEPET}

ADDRESS: Ahi Evran University, Department of Mathematics, 40200, Kirsehir, Turkey.

E-MAIL: sezinaykurt@hotmail.com

ORCID ID : orcid.org/0000-0003-1521-6798

\section{MAHMUT ERGÜT}

ADDRESS: Namik Kemal University, Department of Mathematics, 59000, Tekirdag, Turkey.

E-MAIL: mergut@nku.edu.tr

ORCID ID : orcid.org/0000-0002-9098-8280 